\title{
Economic Traffic Management: Mechanisms and Applications
}

\author{
Fabio Hecht and Burkhard Stiller \\ University of Zürich, Communication Systems Group CSG, Switzerland \\ \{hecht, stiller\}@ifi.uzh.ch
}

\begin{abstract}
The paradigm shift from centralized services into hundreds of thousands of decentralized services changes traffic directions and patterns in the Internet. In that light, overlay providers form logical communication topologies, which effect the underlying telcos' as well as Internet Service Providers' (ISP) traffic and network management. Thus, Economic Traffic Management (ETM) principles deal with incentives, decentralized traffic generation, and modern applications, such as integrated Video-on-Demand and live streaming approaches. Therefore, this work overviews the very recent work on ETM mechanisms, especially driven by the SmoothIT project, and on LiveShift, combining efficiently architectural and system demands for an incentive-driven application management. Furthermore, this section describes in examples, provides evidences, and concludes that ETM and LiveShift can pave an efficient path to modern application support of distributed overlay traffic management, which benefits telcos, ISPs, overlay providers, and end users, if all or parts of them see the right incentives to cooperate, behave well, or interact.
\end{abstract}

Keywords: Economic Traffic Management, distributed overlay traffic management, incentives, $\mathrm{P} 2 \mathrm{P}$, video-on-demand, live streaming.

\section{Introduction}

The Internet traffic is still doubling approximately every 18-month; up to $80 \%$ of this traffic stems from Peer-to-Peer (P2P) and other overlay network-based applications [10]. Since bandwidth and reachability of end-nodes is also increasing, e.g., due to the deployment of Fiber-to-the-Home (FTTH) access, overlay-based applications are expected to continue contributing to a large share of Internet traffic in the future. This indicates that a paradigm shift from services offered centrally to services offered by end-nodes is already happening, and most network traffic will follow network paths forming logical network overlays at the service layer, such as Virtual Private Networks, Network Management System overlays, or distributed collaboration overlays. For today's Telecommunication Service Providers (telco) and Internet Service Providers (ISP) the crucial issue of how to control and manage network traffic stemming from these overlay-based applications arises.

The use of Economic Traffic Management (ETM) mechanisms based on incentives for controlling and managing network traffic of overlay applications in the Internet has 
been researched very recently and is still in its early stages [21]. However, initial results have shown that such mechanisms do have the important property of scalability with respect to the number of players (i.e. providers, consumers, or peers), and also that they do lead to a more efficient network operation. In managing traffic created and routed through their networks, today's ISPs, which may be service providers on the basis of IP (Internet Protocol), employ methodologies suitable for traditional traffic/service profiles. As the structure of overlays determines the traffic flows in ISP networks, it is highly desirable for ISPs to influence the overlay configuration based on information on their specific structure or use. Thus, overlays must be managed to maximize the advantages for multiple ISPs involved (independently of underlying topology), increase their capability to withstand faults, and balance network load.

From a user's perspective, ETM mechanisms show the potential to be applied to a wide range of services [21]. Those services may differ with respect to Quality-of-Service (QoS) metrics and may be charged differently. This enables a market of competitive overlay services and their management. Users will benefit from this competition and the variety of services and service qualities, made conveniently available to users.

SmoothIT [20] has investigated several ETM mechanisms to bridge the gap between overlay and underlay to benefit ISPs, overlay providers, and overlay users [21]. ETM provides the relevant decentralized control technology and management approaches, backed by thorough theoretical investigations, which enable new service quality levels. The application of such scalable ETM mechanisms enables ISPs to reduce their service provisioning and maintenance costs. At the same time, since other overlay applications often cover end-users connected to multiple ISPs, appropriate incentives schemes for collaboration among ISPs (which may also be competing ones at the same time) have been defined and investigated thoroughly. In addition, the incentives need to match detailed application requirements, to be applicable and successful.

Thus, the LiveShift approach determines a P2P application [11] forming an overlay on its own - supporting both live and time-shifted steaming in an integrated manner. It allows for the collaboration beyond the current state of the art, enabling peers to store video streams received in order to distribute them in the future. A highly relevant use case of this functionality allows users, without having previously prepared any local recording, to watch programs from the start or to jump over uninteresting parts until seamlessly catching up with the live stream. Watching pre-recorded or live content does not require different protocols and system architectures - LiveShift provides these two functionalities - , however, up to now only two incompatible incentive schemes exist, which cannot cope with this LiveShift integration of live and time-shifted streams. Thus, LiveShift provides an interesting use case for the deployment of the ETM mechanisms defined, since the feature of storing and a later distributing video streams increases the opportunities of peer collaborations.

The remainder of this section presents in Sect. 2. major related work, which is combined with a discussion of incentives in distributed systems. In support of the feasibility of ETM and an integrated LiveShift streaming system, the two architectures of the system and applications side are defined in Sect. 3.. These development steps are complemented by a selected set of results obtained throughout the respective evaluation in Sect. 4. Finally, Sect. 5. summarizes the key findings and draws conclusions. 


\section{Related Work and Incentives in Distributed Systems}

Related work in the field of ETM and incentives has addressed only very recently this research domain of integrating economically-driven approaches with very specialized optimization tasks. Thus, key approaches are sketched and briefly discussed.

Thus, [13] described early concepts that can be considered to be a very early and limited instance of ETM, which is applied to overlay traffic. Furthermore, the Knowledge Plane [6] defines a system for gathering observations, constraints, and assertions, which applies rules to these to generate observations and responses for network operation. As such it provides a basis for the autonomy in ETM. More specifically, the concept of Biased Neighbor Selection [3] introduces the idea of adapting overlays to explore traffic localization. The Ono plug-in approach [5] implements this concept as part of a P2P file-sharing application, exploiting information obtained indirectly from Content Distribution Network (CDN) servers. In addition, a few approaches have been proposed, which try to facilitate the collaboration between P2P applications and ISPs. The P4P project [22] proposes the presence of an information server, called iTracker, to provide locality information to the overlay. The iTracker may communicate directly with peers or application trackers. A similar approach, the Oracle service [2], runs at the ISP and influences the overlay by ordering potential neighbors by the ISP's preference. The ALTO (Application-Layer Traffic Optimization) IETF Working Group [1] is working on defining a standard for such services provided by ISPs to overlay applications. The area of incentives in distributed systems, particularly for P2P systems, has attracted significant attention in the past. Many approaches are interested in providing incentives for peers to share their resources [19], e.g., upload files or provide content. Incentive schemes, such as Tit-for-Tat [7] and CompactPSH [4], rely on reciprocity, which is challenging in a video straeming system, since the stream is bound to flows within a single direction only (in most cases). A different approach is relying on reports sent by peers [14], possibly having a way to verify those reports [16]. The problem is challenging in LiveShift, since it supports live streaming, in which rate-based incentives are appropriate, and fully integrated video-on-demand, in which peers need to store streams to be provided, thus relying on immediate reciprocation is unsuitable. ETMs go beyond this definition to provide incentives for multiple players to support the distributed service provision. In that sense the alternative choices for ETMs are outlined in further detail in Sect. 3.1 below, since they have been developed in theory and implementation by the SmoothIT project.

\section{$3 \quad$ ETM and Application Architecture}

To reach a suitable approach for meeting the set of requirements as outlined, (a) relevant ETM mechanisms developed during the SmoothIT project and (b) a description of the architectures of the ETM System and LiveShift, as a possible application for the deployed ETM mechanisms, has to be defined. In that respect these areas are outlined below to provide the basis for a subsequent evaluation. 


\subsection{ETM Mechanisms}

To enable overlay traffic management techniques, which include economically-driven incentives, relevant stakeholders' (users, ISPs, and overlay providers) and their evaluations are required to be determined. In that sense so-called "win"-situations define that a stakeholder does see a positive effect upon applying an ETM mechanism. In the best cases, the application of ETM mechanisms lead to a TripleWin situation, in which all three stakeholders are identified to benefit from ETM. The most important of these can be classified into the following three main categories [21]:

Locality promotion involves all peers of a domain, in which they rate overlay neighbors according to their underlay proximity. Within the BGPLoc (border Gateway Protocol Locality) approach a peer sends a list of peers to the SIS (SmoothIT Information Service) [18]. The latter interfaces with the underlay network, obtains locality information, and rates each peer based on certain location attributes, e.g., on the number of hops in the Autonomous System (AS) path. This rated and accordingly sorted list is returned to the requesting peer where dedicated overlay operations (e.g., unchoking and neighbor selection in BitTorrent) are modified to favor peers belonging to the same domain with the requesting one. Thus, interconnection costs for ISPs are reduced, while the performance for peers is also improved in a variety of cases.

With the insertion of locality-promoting peers or resources an indirect way of promoting locality for the ISP is introduced [15] . This is based on a domain-special resource, such that content is downloaded faster into the domain and distributed among local peers. Thus, in IoP-based (ISP-Owned Peer) solutions the IoP initially participates in the swarm as a normal peer, but quickly acquires the entire content and starts serving the local peers. Two instances of such ETM mechanisms have been developed, besides the IoP the Highly Active Peer (HAP). While in case of the IoP the ISP deploys and controls a peer with augmented bandwidth and storage capabilities (allowing for a download of content and making it available to domain's peers), the HAP this idea is implemented in a decentralized manner, with the functionality being passed to normal peers. More specifically, due to overlay and underlay information, certain highly active peers in a domain are promoted by the ISP to so-called enhanced peers and their Internet connection speed is increased dynamically by the ISP, mainly to enable a faster download and to offer overlay content efficiently.

The inter-domain collaboration (Inter-SIS) addresses cases, where information available to a domain is not sufficient to reach some of the aforementioned optimization objectives [8], [9]. This can be due to the asymmetry of routing information between source and destination domains or it is due to the lack of connection details of communicating peers. In such cases, inter-domain collaboration can lead to fine-tuning and improvement of local decisions in all participating domains.

\subsection{SmoothIT's ETM System Architecture}

To provide for a suitable, seamlessly integrated, and efficient architecture for ETM mechanisms in today's and tomorrows Internet Fig. 1 shows the relevant architecture developed to support the overlay and underlay integration through the so-called ETMS (ETM System) [21]. Within the ETMS layer, the SIS (SmoothIT Information Service) typically runs in ISPs premises. 


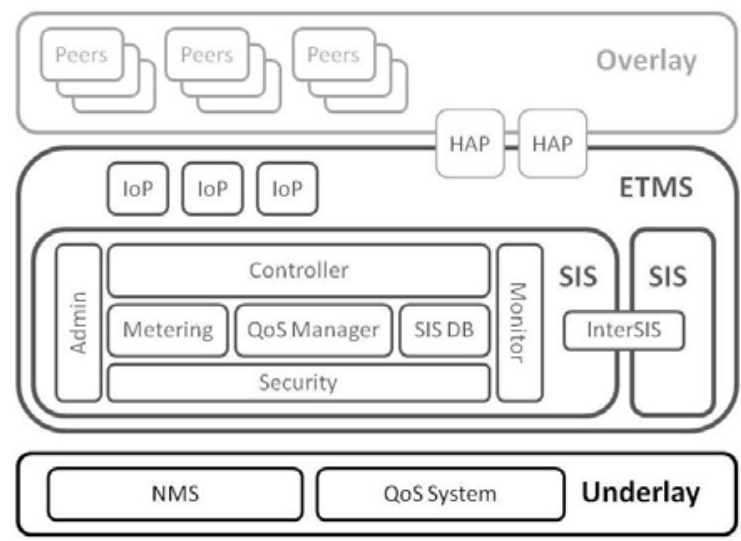

Fig. 1. SmoothIT ETM System Architecture

The Controller component determines the core of the system: it is responsible for the coordination of the ETM mechanism by receiving requests from overlay applications, the calculations based on the ETM mechanism chosen, and in cooperation with several underlay metrics (e.g., provided by metering and policy information) for the output of the required information to the overlay application. This information returned is in form of ranked lists, such as rating peers as possibly "good" neighbors or as rating swarms as "popular" ones.

The Metering component is responsible for collecting network information from the underlying Network Management System (NMS) and supplying them to the ETM mechanisms in the SIS. This information can include, e.g., BGP (Border Gateway Protocol) routing information, network performance parameters, and user profiles. Furthermore, the QoS Manager checks the availability of network resources, and may be used also to guarantee resources requested by the end user, enforcing QoS policies in the network. It interfaces to the network by using, for example, NGN (Next Generation Network) transport control functionalities. Additionally, the InterSIS component facilitates the exchange of information between two or more SISs to fine-tune local decisions. Finally, the SIS DB (Database) stores all information that may be useful for all modules, such as configuration parameters or business logic. Further details can be obtained from [21].

\subsection{LiveShift's Application Architecture}

LiveShift is introduced as a promising example application to fit the ETM architecture as introduced at the overlay layer, since it provides both highly dynamic content (the live stream) and content that can be stored (the distributed storage of past live streams). Using the ETMS on LiveShift does show a great potential, due to the inherent difficulty in providing the right incentives, mainly due to the mixed scenarios of high traffic loads generated and the delay-sensitivity present in video streaming, all of which influencing the quality-of-experience of the end user. LiveShift follows the mesh-pull approach, in 
which peers split video streams into chunks, and exchange them with no fixed structure, using (1) a publish/subscribe protocol to report chunk availability and (2) a distributed tracker for content and peer discovery [11].

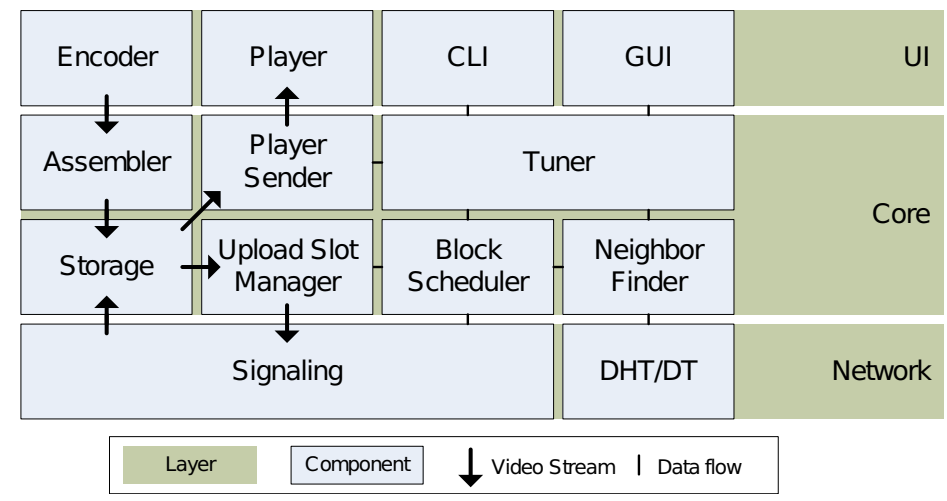

Fig. 2. LiveShift architecture

While Fig. 2 shows major components of the LiveShift architecture, which are split into three layers: the Network layer (which corresponds to the Overlay in Fig. 1) contains the Distributed Tracker (DT) and the Distributed Hash Table (DHT) to store network-wide persistent information in a fully-decentralized manner, and a signaling component to handle direct communication between peers. The DT and DHT are provided by the TomP2P library [2]. The signaling component handles direct communication between peers. Peers use it to send unicast messages to other peers.

The main challenge in LiveShift (and in fact any P2P video streaming system) is to locate and download streams on time to keep up with the playback and with the added challenge of a potentially large time-scale. While incentive mechanisms in their original form (application-centered only) show limitations in providing both rate-based and long-term incentives for peers to share, ETM mechanisms do help in this respect by avoiding congestion, suggesting peers with lower latency or higher available bandwidth, caching content for time-shifting, and further providing incentives for peers to share. Thus, a combination of ETM and LiveShift shows valuable benefits.

\section{$4 \quad$ Evaluation and Results}

Besides the design of this emerging and innovative functionality, the evaluation and suitability needs to be proven, too. Thus, a selection of major results obtained with the introduced ETMS is outlined in Sect. 4.1, focusing on results on the BGPLoc ETM mechanism. And to complete, Sect. 4.2 contains selected LiveShift results, which address the proposed use case from above and which have been derived from an implemented prototype. 


\subsection{ETM Samples}

The ETM system designed has been implemented prototypically [2]. The overlay application used there is based on Tribler, a P2P file-sharing application that implements the BitTorrent protocol. The modified application includes the BGPLoc ETM mechanism, which supports locality promotion at two peer selection algorithms: Biased Neighbor Selection (BNS) prioritizes selecting local peers for downloading, Biased Optimistic Unchoking (BOU) peers prefer local peers to grant their optimistic unchoking slot. For more information on the BitTorrent protocol, refer to [7].

The following results were obtained by simulating one BitTorrent swarm for the distribution of a file with size $154.6 \mathrm{MB}$ for 6.5 hours [18]. The simulated topology consists of 20 Tier 3 stub ASes, two Tier 2 hub ASes, and one Tier 1 transit AS. Each stub, which AS runs an instance of the ETMS and a number of peers, and is directly connected to two other stub-ASes (depicting peering links) and one hub-AS. The two hub-ASes are interconnected via the transit-AS (transit links). Peers join the swarm at a random (uniformly distributed) stub AS with an exponentially distributed inter-arrival time with a mean value of $10 \mathrm{~s}$, and remain online until they have downloaded the entire file plus an additional, exponentially distributed seeding time with a mean value of 10 minutes. In all experiments, four different peer behaviors are compared: regular BitTorrent (regBT), BitTorrent with Biased Unchoking (BOU), Biased Neighbor Selection (BNS), and both (BNS\&BOU) [18].

Additionally, the application supports live monitoring by periodically sending reports to the Monitor component, including overlay statistics (e.g., total download and upload, download and upload rate, or download progress), and also video playback statistics (e.g., play and stall time), which are stored in the SIS Database. All components of the SIS have been implemented in Java and are deployed on a JBoss application server. The current version of the Controller supports the BGP-based locality promotion (BGPLoc) and IoP mechanisms.

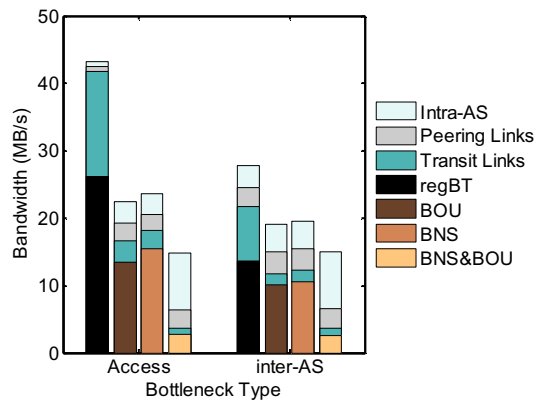

Fig. 3. Mean BitTorrent traffic (ISP) [18]

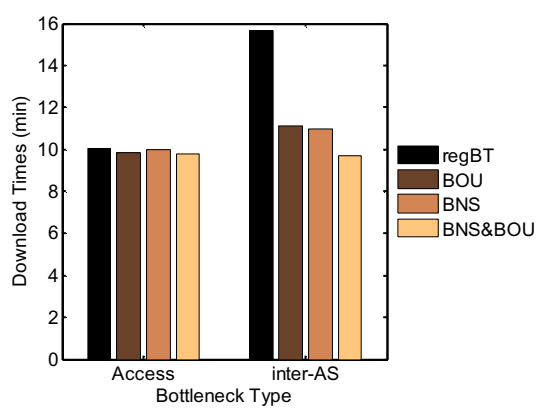

Fig. 4. Mean download time (end user) [18]

Fig. 3 and Fig. 4 consider that the networks suffers from a bottleneck at two different points: while the "Access" bottleneck assumes that there is no congestion in 
peering and transit links, the "Inter-AS" bottleneck considers that traffic shaping takes place between stub and hub ASs. Fig. 3 shows the traffic measured at different link types: the ISP is always able to save costs by reducing traffic at transit links, since intra-AS connections are preferred. Also, these savings are larger with the combination of BNS\&BOU. Fig. 4 shows that download times remain unaffected, if the bottleneck is at the access link, but experiences a worse performance with regBT and inter-AS bottleneck. For results obtained with other ETM mechanisms, especially IoP, Inter-SIS, and HAP, refer to [15], [8], [9], and [17], respectively.

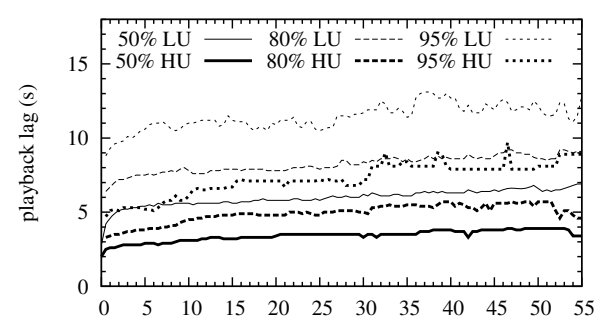

Fig. 5. Experienced playback lag vs. holding time in scenario s1

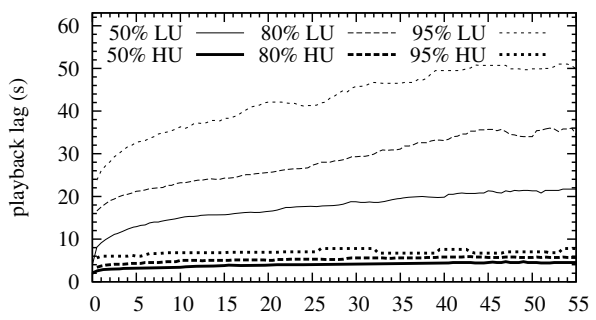

Fig. 6. Experienced playback lag vs. holding time in scenario $\mathrm{s} 3$

\subsection{LiveShift Samples}

LiveShift has been fully implemented in Java 1.6 and evaluated in a test-bed using traces to model user behavior. A complete definition of test-bed, scenarios, and user behavior can be found at [11]. A number of users were emulated, switching among 6 channels, and reporting metrics such as their playback lag or channel holding times.

Fig. 5 shows the experienced playback lag for scenario s1, which is composed out of 6 peercasters (peers that are sources for a channel, can upload 5 times the video stream), 15 high upstream peers (HU, can upload 5 times the video stream), and 60 low upstream peers (LU, can upload 0.5 times the video stream). Scenario s1, being well-provisioned with bandwidth, shows a low (i.e. less than 15s) playback lag for sessions of up to one hour for $95 \%$ of all HU and LU peers. Fig. 6 outlines values for Scenario s3, which, in contrast with s1, has 120 LU peers, thus, being on the edge of being underprovisioned in terms of upload capacity. Although the playback lag is considerably higher than on s1, which is natural, considering that it will take longer for peers to find available bandwidth, and the average hop count that every chunk needs to travel is higher, on average. The playback lag in s3 remains below $60 \mathrm{~s}$ for $95 \%$ of all peers.

Fig. 7 shows that (a) the overhead introduced by LiveShift is small (less than $3 \%$ ), since the video stream it transports is already very bandwidth-consuming, and (b) that in Scenario s3 the share of failed playbacks (that is, the user received less than half of all video blocks in a moving window of $30 \mathrm{~s}$ and gave up) is already larger than $4 \%$. 

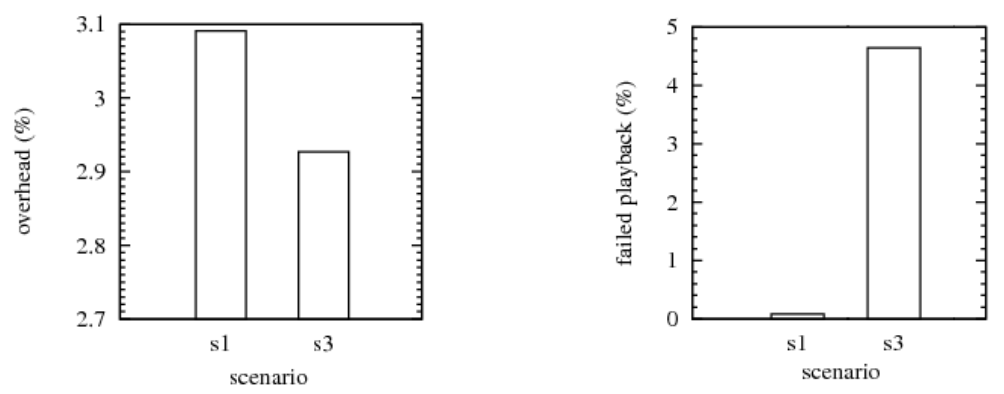

Fig. 7. Overall overhead and average failed playback in scenarios s1 and s3

\section{Summary and Conclusions}

This section describes a set of ETM (Economic Traffic Management) mechanisms defining alternatives to promote (directly or indirectly) localization of overlay traffic, namely the BGP-Loc, the IoP, and the Inter-SIS mechanisms. In combination with the LiveShift application, such modern overlays and respective functionality can support a scalable and distributed traffic and application management in the Internet.

To enlighten the feasibility of these approaches introduced, designed, and discussed, the respective architectures of the ETM System and LiveShift have been deployed in terms of a practical implementation performed in the SmoothIT and LiveShift approaches. Also, the key concepts, the use case, and evaluation results as presented show their potential in profiting from the presented ETM mechanisms and related incentives. In that sense, these approaches are backed by selected results from test-bed trials and performed evaluations.

Concluding, the application of economically-driven incentive mechanisms in a distributed networking environment show besides their implementation feasibility the advantages for different stakeholders under different overlay set-ups. In this variety of evaluations the promotion of locality information is highly beneficial, while avoiding the violation of any stakeholders' own optimization (TripleWin). Especially and in case of video sharing via BitTorrent, the combination of different peer behaviors show winning mechanisms, which is reachable by an flexible architectural support. Additionally, the behavior of the streaming/Video-on-Demand approach LiveShift indicates that acceptable playback delay are achievable, which does lead to valuable user experience. This is complemented by the fact that also for LiveShift the overhead introduced is very small, e.g., in terms of failed playback delays. In turn, the (a) feasibility of those approaches, (b) the technical integration seamlessly into existing networking infrastructures, and (c) the achieved technical performance indicate that the ETM key idea combined with the incentives aspect show a beneficial outcome for overlay management principles.

Acknowledgement. This work has been performed partially in the framework of the EU Project SmoothIT (FP7-2007-ICT-216259), the COST Action IS0605, the EU Project SESERV (FP7-2010-ICT-258138), and the project LiveShift at CSG@IFI. The 
authors like to acknowledge particularly T. Hoßfeld, S. Oechsner, F. Lehrieder, K. Pussep, M. Michel, S. Soursos, I. Papafili, G. Stamoulis for providing partial results, and all members of SmoothIT and SESERV for their collaboration and support.

Open Access. This article is distributed under the terms of the Creative Commons Attribution Noncommercial License which permits any noncommercial use, distribution, and reproduction in any medium, provided the original author(s) and source are credited.

\section{References}

1. Application-layer traffic optimization (alto), IETF Working Group (2009), http: / /www. ietf.org/html. charters/alto-charter.html

2. Aggarwal, V., Feldmann, A., Scheideler, C.: Can ISPs and P2P systems co-operate for improved performance? ACM Computer Communication Review 37(3), 29-40 (2007)

3. Bindal, R., Cao, P., Chan, W., Medval, J., Suwala, G., Bates, T., Zhang, A.: Improving Traffic Locality in BitTorrent via Biased Neighbor Selection. In: 26th IEEE International Conference on Distributed Computing Systems, Lisbon, Portugal, p. 66 (July 2006)

4. Bocek, T., Hecht, F.V., Hausheer, D., Stiller, B., El-khatib, Y.: CompactPSH: An efficient transitive TFT incentive scheme for Peer-to-Peer Networks. In: 34th Annual IEEE Conference on Local Computer Networks (LCN 2009), Zürich, Switzerland, pp. 483-490 (October 2009)

5. Choffnes, D.R., Bustamante, F.E.: Taming the Torrent: A Practical Approach to Reducing Cross-ISP Traffic in Peer-to-peer Systems. ACM Computer Communication Review 38(4), 363-374 (2008)

6. Clark, D., Partridge, C., Ramming, J., Wroclawski, J.: A Knowledge Plane for the Internet. ACM Computer Communication Review 33(4) (October 2003); ACM SIGCOMM, Karlsruhe, Germany, pp. 3-10

7. Cohen, B.: Incentives Build Robustness in BitTorrent. In: 1st Workshop Economics of Peer-to-Peer Systems (P2PCON), Berkeley, California, U.S.A. (June 2003)

8. Dulinski, Z., Stankiewicz, R., Wydrych, P., Cholda, P., Stiller, B.: Inter-ALTO Communication Protocol. IETF Internet-Draft, draft-dulinski-alto-inter-alto-protocol-00 (June 2010); work in progress

9. Dulinski, Z., Wydrych, P., Stankiewicz, R.: Inter-ALTO Communication Problem Statement. IETF Internet-Draft draft-dulinski-alto-inter-problem-statement-01 (July 2011); work in progress

10. Haßlinger, G.: ISP Platforms Under a Heavy Peer-to-Peer Workload. In: Steinmetz, R., Wehrle, K. (eds.) P2P Systems and Applications. LNCS, vol. 3485, pp. 369-381. Springer, Heidelberg (2005)

11. Hecht, F.V., Bocek, T., Clegg, R.G., Landa, R., Hausheer, D., Stiller, B.: LiveShift: Mesh-Pull Live and Time-Shifted P2P Video Streaming. In: 36th IEEE Annual Conference on Local Computer Networks (LCN 2011), Bonn, Germany, pp. 319-327 (October 2011)

12. Lehrieder, F., Oechsner, S., Hoßfeld, T., Despotovic, Z., Kellerer, W., Michel, M.: Can P2P-Users Benefit from Locality-Awareness? In: 10th IEEE International Conference on Peer-to-Peer Computing 2010 (P2P 2010), Delft, The Netherlands (August 2010)

13. Liu, Y., Zhang, H., Gong, W., Towsley, D.: On the Interaction between Overlay Routing and Underlay Routing. In: INFOCOM 2005, Miami, Florida, U.S.A., pp. 2543-2553 (2005)

14. Mol, J.J.D., Pouwelse, J.A., Meulpolder, M., Epema, D.H.J., Sips, H.J.: Give-to-get: Free-riding-resilient video-on-demand in $\mathrm{p} 2 \mathrm{p}$ systems. Multimedia Computing and Networking 6818, 681804-681804-8 (2008) 
15. Papafili, I., Stamoulis, G.D.: A Markov Model for the Evaluation of Cache Insertion on Peer-to-Peer Performance. In: EuroNF NGI Conference, Paris, France (June 2010)

16. Piatek, M., Krishnamurthy, A., Venkataramani, A., Yang, R., Zhang, D., Jaffe, A.: Contracts: Practical Contribution Incentives for P2P Live Streaming. In: 7th USENIX Conference on Networked Systems Design and Implementation, Berkeley, California, U.S.A., September 2010, p. 6 (2010)

17. Pussep, K.: Peer-Assisted Video-on-Demand: Cost Reduction and Performance Enhancement for Users, Overlay Providers, and Network Operators; Ph.D. Thesis, Technische Universität Darmstadt, Germany (2011)

18. Racz, P., Oechsner, S., Lehrieder, F.: BGP-Based Locality Promotion for P2P Applications. In: 19th International Conference on Computer Communications and Networks (ICCCN 2010), Zürich, Switzerland, pp. 1-8 (August 2010)

19. SESERV Project (October 2011), http: / / www . seserv . org

20. SmoothIT Project (March 2011), http: / /www . smoothit.org

21. Soursos, S., Callejo Rodriguez, M., Pussep, K., Racz, P., Spirou, S., Stamoulis, G., Stiller, B.: ETMS: A System for Economic Management of Overlay Traffic. In: Tselentis, G., et al. (eds.) Towards the Future Internet — Emerging Trends from European Research, pp. 1-10. IOS Press, Amsterdam (2010)

22. Xie, H., Yang, R.Y., Krishnamurthy, A., Liu, Y.G., Silberschatz, A.: P4P: Provider Portal for Applications. ACM Computer Communication Review 38(4), 351-362 (2008) 